\title{
Anatomical Study on the Variations in the Branching Pattern of Internal Iliac Artery
}

\author{
H. Mamatha $\cdot$ B. Hemalatha $・$ P. Vinodini • \\ Antony Sylvan D. Souza $\cdot$ S. Suhani
}

Received: 14 June 2012 / Accepted: 21 November 2012 /Published online: 20 December 2012

(C) Association of Surgeons of India 2012

\begin{abstract}
The internal iliac artery (IIA) arises from the common iliac artery at the level of the sacroiliac joint. It descends as trunk, divides into anterior and posterior divisions. From the anterior division it gives superior vesical, obturator, middle rectal, inferior vesical, inferior gluteal, and internal pudendal arteries. In females, inferior vesical artery is replaced by vaginal artery and gives an additional branch, uterine artery. The branches from the posterior division are ilio lumbar, lateral sacral, and superior gluteal arteries. An accidental hemorrhage and neurovascular injuries are common due to erroneous interpretation of variant arteries during surgical procedures. Hence the present study has been undertaken with reference to highlight its clinical and surgical significances. The study included 50 human bisected pelvises irrespective of their side and sex. Formalin-fixed specimens were selected and the branching patterns of IIA were studied. The level of origin, length, and the branching pattern of the IIA were examined and tabulated. The present study highlighted the variation seen in the origin, division branches of the IIA. The detailed knowledge of morphology is essential for successful ligation of the IIA during acute hemorrhage. This will guide the interventional radiologist in intra-arterial procedures during arterial embolization for hemorrhage, control of pelvic fractures, during selective catheterization of the intraarterial chemotherapy, and embolization of the pelvic tumors.
\end{abstract}

Keywords Internal iliac artery - Accidental hemorrhage ·

Arterial ligations $\cdot$ Embolization

\section{Abbreviations \\ IIA Internal iliac artery \\ OA Obturator artery}

H. Mamatha • B. Hemalatha • P. Vinodini - A. S. D. Souza •

S. Suhani $(\bowtie)$

Department of Anatomy, Kasturba Medical College,

Manipal University, Manipal 576104 Karnataka, India

e-mail: contact_suhan@yahoo.co.in

\section{Introduction}

The IIA originates from the common iliac artery at the level of sacroiliac joint, descends around the upper border of greater sciatic foramen, and divides into anterior and posterior divisions. The branches of the anterior division include superior vesical artery, inferior vesical artery, middle rectal artery, vaginal artery, obturator artery (OA), internal pudendal artery, and the inferior gluteal arteries and provide territorial supply to the pelvic viscera $[1,2]$.

The posterior division has three branches such as iliolumbar artery, lateral sacral artery, and the superior gluteal artery, and distributes to the body wall and buttocks. In general, the IIA supplies the pelvic viscera, the pelvis walls, the perineum, and the gluteal region $[3,4]$.

During the development, the IIA derives from the umbilical artery, which persists in its proximal part and the distal part gets obliterated postnatally. However, due to embryological modifications, variations in the vascular pattern of the IIA were frequently quoted. Such incidences create an eye opener for the gynecologists and surgeons during intra-abdominal therapeutic procedures. The detailed knowledge and its branching pattern of the IIA will provide the surgeons a wide access to the guidelines in ligating the artery and its branches during various surgical procedures of the pelvic viscera [5]. Hence the present study has been undertaken with reference to the morphological significance of the IIA.

\section{Materials and Methods}

The study included 50 human cadaveric bisected pelvises during the routine dissection in the Department of Anatomy KMC, Manipal. Formalin-fixed specimens were selected irrespective of their side and sex and the branching pattern of the IIA were extensively studied. 
Table 1 Origin of internal iliac artery at various levels

\begin{tabular}{lcc}
\hline Vertebral level & No. of specimen & Percentage \\
\hline L5-S1 vertebra & 36 & 72 \\
S1 vertebra & 12 & 24 \\
L5 vertebra & 2 & 4 \\
\hline
\end{tabular}

The IIA, its anterior and posterior divisions and its subdivisions, were meticulously dissected and painted, separating from the surrounding structures, and after drying they were photographed.

The objectives of our study were to observe the vertebral level of origin of the IIA and to determine the level of the division and the branching patterns of the IIA.

\section{Results}

Table 1 illustrates the origin of the IIA at various levels. The origin of the IIA at the level of S1 vertebra was seen in 36 cases (72\%), at the level of L5-S1 in 12 cases (24\%), and in two cases $(4 \%)$. The distance of the level of division of the IIA in respect to greater sciatic foramen is shown in Table 2. The distance from the greater sciatic notch is $3 \mathrm{~cm}$ above in nine cases (18\%), $2 \mathrm{~cm}$ above in $14(28 \%)$ cases, $2.5 \mathrm{~cm}$ above in 18 specimens (36\%), $3.5 \mathrm{~cm}$ above in eight cases (16 $\%)$, and $1 \mathrm{~cm}$ below the greater sciatic notch in one case (2\%). Table 3 shows the variations in branching pattern of the IIA like the OA branching from the posterior division of the IIA (Fig. 1), abnormal OA branching from the inferior epigastric artery (Fig. 2), iliolumbar, direct branch from the trunk of the IIA (Fig. 3), OA branching from the posterior division of the IIA (Fig. 4), and OA branching with the inferior vesical artery (Fig. 5).

\section{Discussion}

According to Williams et al. and Naveen et al., the IIA arises at the level of the L5-S1 and S1 intervertebral disk and anterior to the sacroiliac joint. However, in the present study the origin of the IIA was at the level of L5-S1 in majority of

Table 2 Distance from greater sciatic notch

\begin{tabular}{lcc}
\hline Distance & No. of specimens & Percentage \\
\hline Above $2 \mathrm{~cm}$ & 14 & 28 \\
Above $2.5 \mathrm{~cm}$ & 18 & 36 \\
Above $3 \mathrm{~cm}$ & 9 & 18 \\
Above $3.5 \mathrm{~cm}$ & 8 & 16 \\
Below $1 \mathrm{~cm}$ & 1 & 2 \\
\hline
\end{tabular}

Table 3 Variation in branching pattern of IIA

\begin{tabular}{lcr}
\hline $\begin{array}{l}\text { Variation } \\
\text { in branches }\end{array}$ & $\begin{array}{l}\text { No. of } \\
\text { specimens }\end{array}$ & Percentage \\
\hline No of variant branch & 30 & $60 \%$ \\
Iliolumbar from trunk & 3 & $6 \%$ \\
Obturator from posterior division & 7 & $14 \%$ \\
Obturator with inferior vesical artery & 4 & $8 \%$ \\
Abnormal obturator artery & 6 & $12 \%$ \\
\hline
\end{tabular}

the specimens. It was reported that the level of origin of the IIA is variable, depending on the length of the common iliac artery and the level of division of the IIA $[1,6]$.

According to Williams et al., the IIA divides at the level of superior edge of greater sciatic notch. In the present study it has been observed that the IIA divided $2-3.5 \mathrm{~cm}$ proximal to the greater sciatic notch in majority of the cases as similar to the study done by Naveen et al. $[1,6]$.

During the development, the IIA derives from the umbilical artery, which persists in its distal part, gets obliterated postnatally. Most appropriate channels enlarge with the others retracting or disappearing, which results in final arterial pattern. Recent report opinions that efficacy of the IIA ligation during any obstetrics and gynecology surgeries varies between 42 and $75 \%[4,5]$.

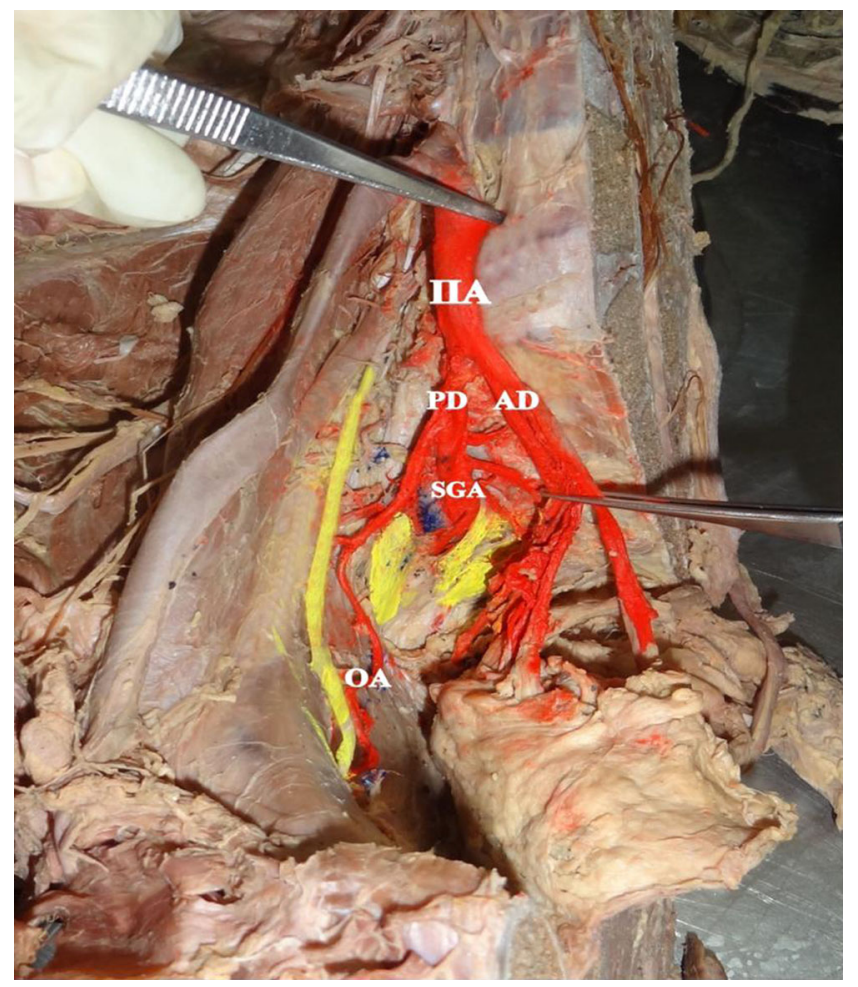

Fig. 1 Obturator artery branching from the posterior division of internal iliac artery. IIA internal iliac artery, $P D$ posterior division, $A D$ anterior division, $S G A$ superior gluteal artery, $O A$ obturator artery 


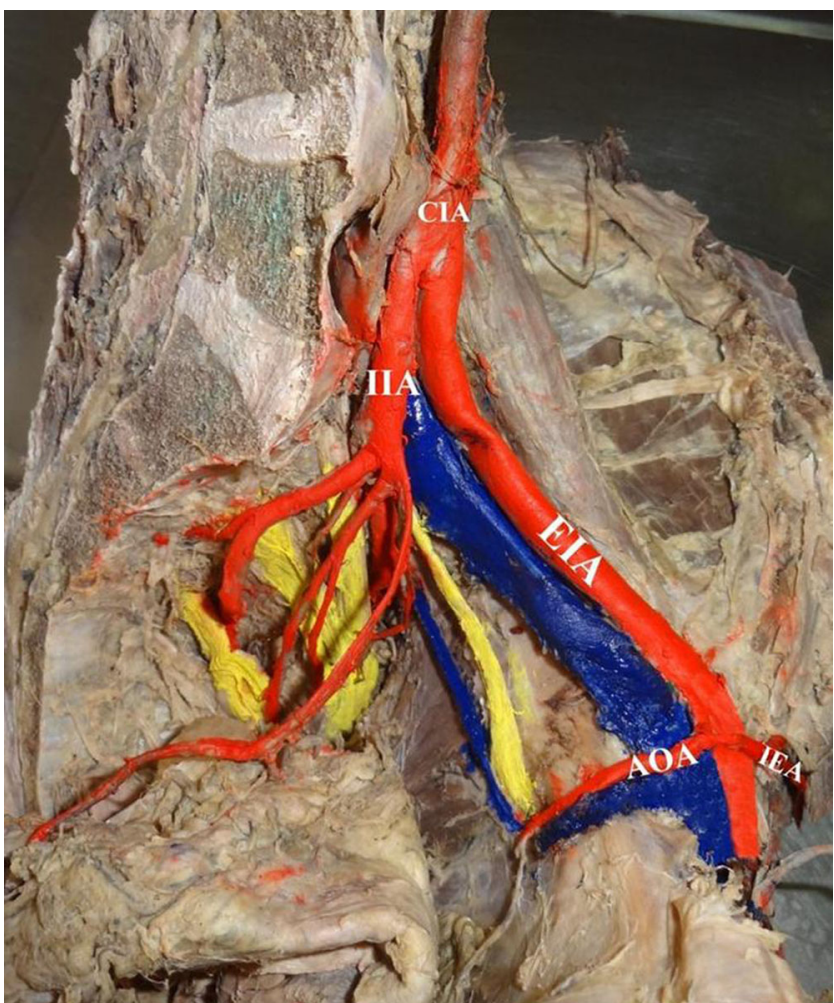

Fig. 2 Abnormal obturator artery branching from inferior epigastric artery. CIA common iliac artery, EIA external iliac artery, IIA internal iliac artery, $A O A$ accessory obturator artery, IEA inferior epigastric artery

So for a successful ligation of the IIA, the operating surgeon should be cognizant of the level of origin, the level of division of the artery; unilateral or bilateral ligation of the IIA can be lifesaving in patients with massive postpartum hemorrhage, after vaginal and abdominal hysterectomy; in massive broad ligament hematoma, cervical carcinoma, and retroperitoneal bleeding after pelvic fractures, bilateral IIA ligation is done. In the present study, in $40 \%$ specimens, variant branches were observed [6-8].

Yamaki et al. [8], in their study based on the dissection of 645 pelvic halves of Japanese cadavers, the branching of the IIA was classified into four groups. In almost $80 \%$ of the specimens, the IIA is dividing into two major branches, the superior gluteal artery and the common trunk for the inferior gluteal and internal pudendal arteries [8].

Ashley and Anson in their study from 130 specimens observed the inferior and superior gluteal arteries were arising from one common stem in $58 \%$ of the cases, the internal pudendal and umbilical arteries were arising from other common stem in $17 \%$ of the cases, the inferior and superior gluteal arteries from one common stem, the internal pudendal and umbilical arteries from other common stem in $8 \%$ of the cases, the umbilical and internal pudendal arteries arose separately above the common stem for gluteal artery [9].

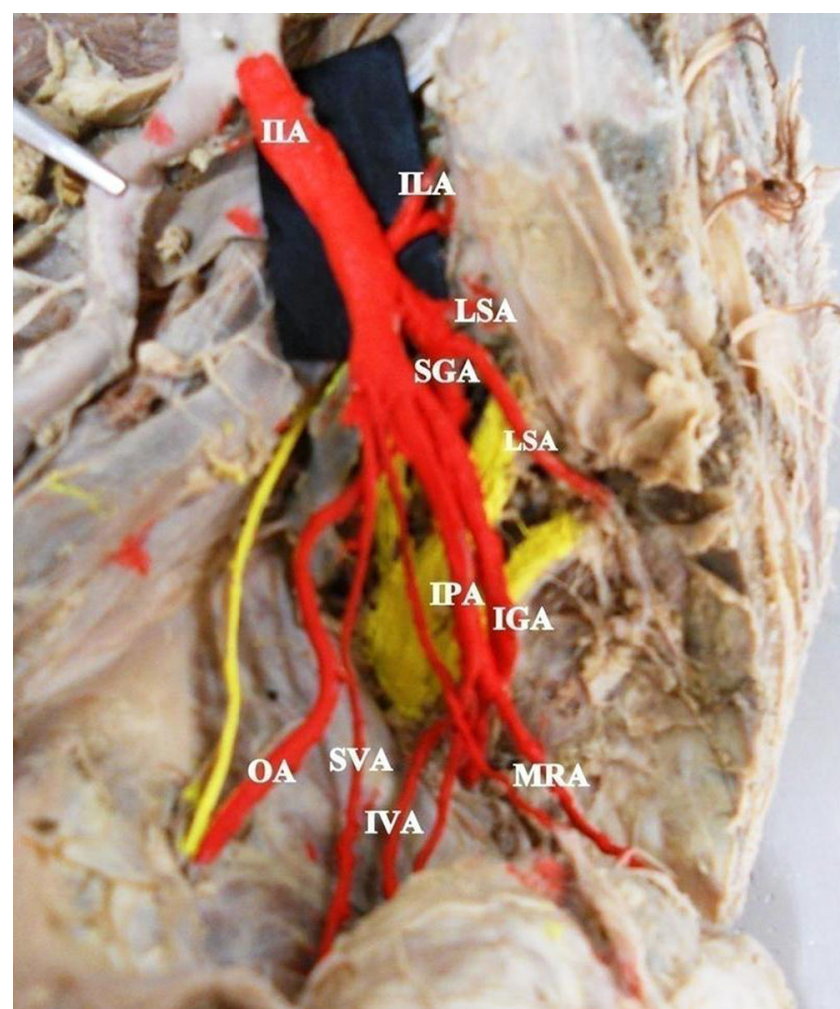

Fig. 3 Ilio lumbar artery, direct branch from the trunk of internal iliac artery. IIA internal iliac artery, ILA ilio lumbar artery, SGA superior gluteal artery, $I P A$ internal pudendal artery, $I G A$ inferior gluteal artery, $M R A$ middle rectal artery, $S V A$ superior vesicular artery, $O A$ obturator artery, IVA inferior vesicular artery

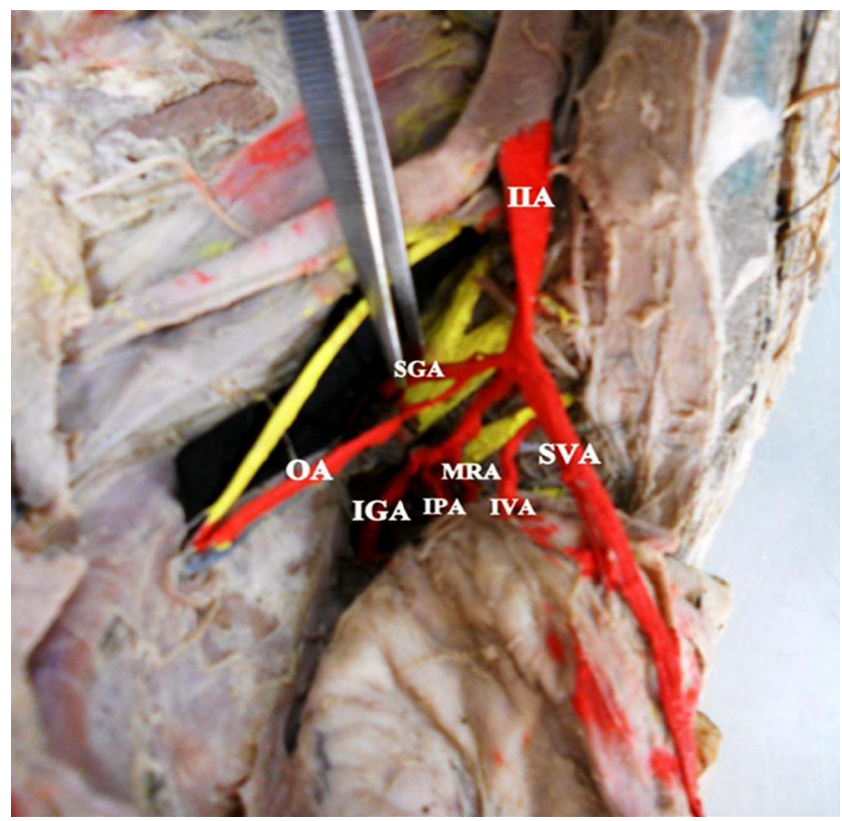

Fig. 4 Obturator artery branching from the posterior division of internal iliac artery. IIA internal iliac artery, $S G A$ superior gluteal artery, $S V A$ superior vesicular artery, $M R A$ middle rectal artery, $I V A$ inferior vesicular artery, IPA internal pudendal artery, $I G A$ inferior gluteal artery, $O A$ obturator artery 


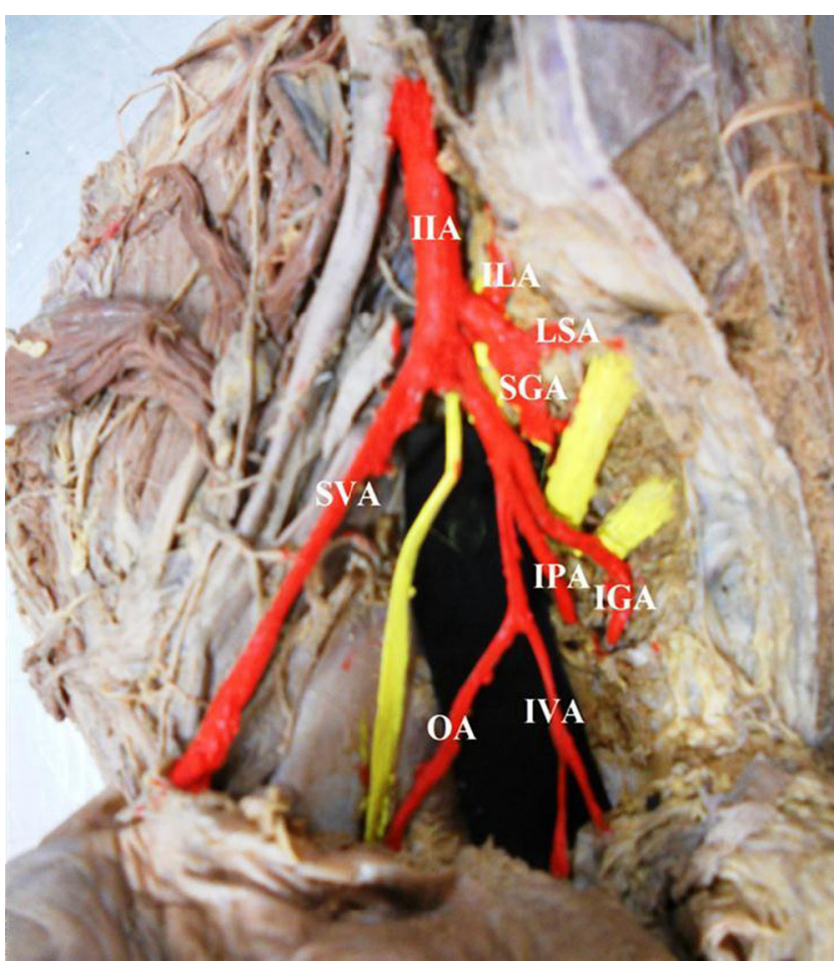

Fig. 5 Obturator artery branching with inferior vesical artery as a common trunk from anterior division of IIA. IIA internal iliac artery, ILA ilio lumbar artery, $L S A$ lateral sacral artery, SGA superior gluteal artery, SVA superior vesicular artery, $O A$ obturator artery, IVA inferior vesicular artery, $I G A$ inferior gluteal artery, IPA internal pudendal artery

However, the findings of our study do not coincide with the previously cited studies. The vascular variations we encountered are as follows: the OA branching from the posterior division of the IIA, abnormal OA branching from inferior epigastric artery, iliolumbar direct branch from the trunk of the IIA, OA branching from the posterior division of the IIA, OA branching with inferior vesical artery.

The OA supplies the hip joint, i.e., head of the femur. In the event of the OA arising from the posterior division of the IIA may be spared during injury to the anterior division and this may be kept in mind during any possible bypass grafting in cases of ischemic necrosis of the head of the femur [10].

Ideally, one would ligate the IIA, distal to its posterior division because proximal ligation has been associated with buttocks claudication and necrosis. Obviously, an anomalous $\mathrm{OA}$ arises from the posterior division as seen in the present study; any ligation of OA would result in erroneous interpretations of angiograms $[10,11]$.

In the present study, the inferior vesical artery was seen arising from the OA (anterior division), inferior vesical, majorly supplies the prostate gland. Therefore, iatrogenic injuries and its consequences must be considered during operative procedures of the pelvis [11].

In the present study, abnormal obturator artery was found arising from the inferior epigastric artery. It lies along the medial margin of lacunar ligament, which possesses an alarming threat during hernia surgeries, wherein it is likely to be cut, when an attempt is made during the routine enlargement of the femoral ring. Unusual selection of channels from the primary capillaries is thought to account for the anomalies affecting the arterial patterns [11, 12].

In life-threatening surgery or delayed intervention to control massive hemorrhage, accidental damage to a ureter, which usually lies on the pelvic surface of the IIA, should be avoided to prevent ureter injuries. Prompt diagnosis and remedial surgery are mandatory. Conditions that indicate ligation as a prophylactic measure include post abortion bleeding, postpartum hemorrhage, abdominal ectopic pregnancies, etc. So general surgeons dealing with laparoscopic herniorrhaphy should be aware of the abnormal obturator artery, crossing superior pubic ramus, which is more susceptible to injuries during mesh stapling into lacunar Cooper ligament [12].

\section{Conclusion}

The present study highlighted the variation seen in the origin, and the divisions of its branches. The detailed knowledge of morphology of IIA is essential for successful ligation of IIA during acute hemorrhage. This will guide the interventional radiologist in the intra-arterial procedures.

Accidental hemorrhage is common during erroneous interpretation of anomalous branching patterns. Life-threatening postpartum hemorrhages have been considered to be the leading cause of maternal deaths in all the developing countries of the world. Thus a thorough knowledge of normal and abnormal anatomy of the branches of IIA is essential for successful ligation of IIA. The ligation of IIA is executed to control hemorrhage during pelvic surgeries, where no definitive bleeding point is detectable. Ignorance about these IIA variations can threaten the patient's life, particularly in the context of pre-existing major blood loss from postpartum hemorrhage.

\section{References}

1. Williams PL, Bannister LH, Berry MM, Collins P, Dyson M, Dussek JE, Ferguson MWJ (1995) Gray's anatomy: the anatomical basis of medicine and surgery, 38th edn. Churchill Livingstone, Edinburgh, p 1560

2. B-Lynch CC, Keith LG, Campbell WB (2006) Internal iliac (hypogastric) artery ligation. A comprehensive textbook of postpartum hemorrhage. An essential clinical reference for effective management, 2nd ed, Sapiens publisher

3. Snell RS (2000) Clinical anatomy for students, 6th edn. Lippincott, William \& Wilkins, Philadelphia, pp 292-293

4. Hollinshed H (1971) Text book of anatomy for surgeons, vol 2, 2nd ed. New York, Harper and Row, pp 217681-217685 
5. Sadler TW (1990) Langman's medical embryology, 6th edn. Williams \& Wilkins Co, London

6. Naveen et al (2011) Morphological analysis of the human internal iliac artery in South Indian population. Online J Health Allied Sci 10(1):11

7. Kelly HA (1894) Ligation of both internal iliac arteries for hemorrhage in hysterectomy for CA uteri. Online J Health Allied Sci 10:53-54

8. Yamaki K, Saga T et al (1998) A statistical study of the branching pattern of the human internal iliac artery. Kurume Med J 45:333-340
9. Ashley FL, Anson BJ (1941) Hypogastric artery in Ametical Whites and Negroes. Am J Phys Anthropol 28:381-385

10. Fatu C, Puisoru M, Fatu IC (2006) Morphometry of the internal iliac artery in different ethnic groups. Ann Anat 188:541-546

11. Usha B, Indu K (2008) Recent advances in the management of postpartum hemorrhage. JK Sci 10(4):163-166

12. Pai MM et al (2009) Variability in the origin of obturator artery. Clin J 64(9):897-901 